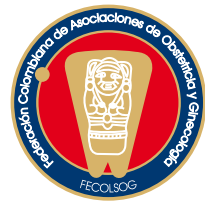

Reporte De CASO

\title{
COMPLICACIONES OBSTÉTRICAS TRAS UNA TRAQUELECTOMÍA RADICAL: CASO CLÍNICO Y REVISIÓN DE LA LITERATURA
}

\author{
Obstetric complications after a radical \\ trachelectomy: case report and review of literature \\ María de la Calle Fernández-Miranda, Ph.D, M.D.*, José Ángel García-Hernández, \\ M.D.**, Ramón Usandizaga Elio***, Javier de Santiago García, Ph.D., M.D.****, \\ Fernando Magdaleno Dans, M.D.*****, Antonio González-González****** \\ Recibido: marzo 24/10 - Aceptado: septiembre 8/10
}

\section{RESUMEN}

Introducción: la traquelectomía radical (TR) es una técnica quirúrgica utilizada en estadios precoces del cáncer de cérvix en aquellas mujeres que desean conservar la capacidad reproductiva. Los embarazos posteriores a esta técnica quirúrgica pueden desencadenar complicaciones obstétricas. El presente artículo tiene como objetivo hacer una revisión de la literatura en relación al pronóstico obstétrico de estas pacientes.

Metodología: en este estudio se presenta un caso clínico. Posteriormente, se realiza una búsqueda en las bases de datos MEDLINE, vía PubMed, y Cochrane con las palabras clave "cáncer de cérvix", "traquelectomía radical”, "embarazo" y "complicaciones obstétricas”.

* Profesor Asociado, Facultad de Medicina, Universidad Autónoma de Madrid. Unidad de Tocología de Alto Riesgo del Servicio de Obstetricia y Ginecología, Hospital La Paz. Madrid (España). Correo electrónico: mdelacalle55@hotmail.com

** Residente de cuarto curso, Servicio de Obstetricia y Ginecología, Hospital La Paz. Madrid (España).

*** Profesor Titular, Facultad de Medicina, Universidad Autónoma de Madrid. Jefe de Sección, Unidad de Suelo Pélvico del Servicio de Obstetricia y Ginecología, Hospital La Paz. Madrid (España).

***** Profesor Asociado, Facultad de Medicina, Universidad Autónoma de Madrid. Jefe de Servicio de Ginecología Oncológica y de Quirófano de Ginecología, Hospital La Paz. Madrid (España).

****** Profesor Asociado, Facultad de Medicina, Universidad Autónoma de Madrid. Jefe de Sección de paritorio del Servicio de Obstetricia y Ginecología, Hospital La Paz. Madrid (España).

******* Catedrático, Facultad de Medicina, Universidad Autónoma de Madrid. Jefe de Servicio de Obstetricia y Ginecología, Hospital La Paz. Madrid (España).
Resultados: se evaluó un total de 17 artículos que comprendieron revisiones, artículos de opinión y casos clínicos.

Conclusión: la preservación de la fertilidad en estadios precoces del cáncer de cérvix mediante la traquelectomía radical está adquiriendo cada vez mayor aceptación a medida que se publican más casos en la literatura. Los resultados obstétricos parecen ser cada vez más favorables aunque son más numerosas las complicaciones en comparación con la población general.

Palabras clave: cáncer de cérvix, traquelectomía radical, embarazo, complicaciones obstétricas.

\section{SUMMARY}

Introduction: radical trachelectomy (TR) is a surgical technique which is used in the early stages of cervical cancer in females wishing to conserve their reproductive ability. Pregnancies following this surgical technique having been applied could trigger obstetric complications. This article was thus aimed at reviewing the literature related to these patients' obstetric prognosis.

Methodology: a clinical case is presented. A literature search was made in MEDLINE databases, via PubMed and Cochrane, using the following key words: "cervical cancer", "radical trachelectomy", "pregnancy", "obstetric complications". 
Results: 17 articles were evaluated, covering review articles, articles expressing opinion and clinical cases.

Conclusion: preserving fertility in early stages of cervical cancer by radical trachelectomy is increasingly acquiring more acceptance as more cases are published in the literature. Obstetric results seem to be becoming more favorable even though complications are becoming more numerous compared to incidence in the general population.

Key words: cervical cancer, radical trachelectomy, pregnancy, obstetric complications.

\section{INTRODUCCIÓN}

El cáncer del cuello del útero es el segundo cáncer más frecuente en la población femenina después del cáncer de mama puesto que afecta anualmente a unas 493.000 mujeres en el mundo y ocasiona la muerte a 274.000 de ellas. ${ }^{1}$ Es una enfermedad que aparece en mujeres en edad reproductiva, por lo que la terapia convencional en estadios tempranos, consistente en una histerectomía radical, conlleva la imposibilidad de gestaciones posteriores en estas pacientes. ${ }^{2}$ La cirugía clásica del cáncer de cérvix incluye la extirpación del cuerpo y el cuello del útero, la resección total de los parametrios y del tercio superior de la vagina, seguida por una linfadenectomía pélvica completa. Sin embargo, los estudios más recientes indican que las pacientes jóvenes, menores de 40 años, con cáncer de cérvix en estadio I pueden beneficiarse de la traquelectomía radical (TR) para conservar su fertilidad. ${ }^{3-6}$

La TR es una técnica quirúrgica desarrollada en 1994 por Dargent, en la que se extirpa el cuello uterino, preservando el cuerpo del útero en los casos de cáncer de cérvix en estadios precoces (estadios de la FIGO IA2-IB1) en aquellas mujeres que deseen preservar su fertilidad. ${ }^{4}$ La TR consiste en una extirpación del cérvix del útero por vía vaginal o abdominal, dejando en algunas ocasiones al menos $1 \mathrm{~cm}$ de cérvix con el fin de disminuir las pérdidas fetales del segundo trimestre en gestaciones posteriores a la cirugía. Se acostumbra dejar catéteres uterinos para drenaje por vía vaginal. ${ }^{4,6-10}$ Previamente a la TR, se realiza una linfadenectomía pélvica por laparoscopia ${ }^{10}$ ya que, si los ganglios pélvicos son positivos, no se realiza la TR. ${ }^{4}$ Debido a sus mejores resultados posoperatorios, la técnica más empleada actualmente es la TR vaginal. ${ }^{8,9}$ Los resultados oncológicos en cuanto a recurrencia y supervivencia libre de enfermedad tras la TR son similares a los de aquellas pacientes a las que se les realiza una histerectomía radical. ${ }^{3-7}$ No obstante, existe controversia sobre las tasas de infertilidad tras una TR y en cuanto al pronóstico. ${ }^{8-12}$

A continuación, se presenta el primer caso publicado en una revista de habla castellana con el objetivo hacer una revisión de la literatura acerca del pronóstico obstétrico posterior a la traquelectomía radical.

\section{PRESENTACIÓN DEL CASO}

Se trata de una paciente de 31 años, nulípara, que acude a urgencias de ginecología del hospital por sangrados vaginales intermenstruales de seis meses de duración. En la exploración vaginal se observa una formación polipoidea de $0,5 \mathrm{~cm}$ en el canal endocervical. Acto seguido, se cita a la paciente en la Unidad de Patología Cervical a los dos días de su visita a urgencias. En la consulta, se le realiza una citología triple toma que se informa como carcinoma poco diferenciado, probable adenocarcinoma. La colposcopia realizada en la misma visita describe una formación polipoidea de $0,5 \mathrm{~cm}$ de características sospechosas con varias atipias sobre la ectopia. La biopsia del pólipo informa que se trata de un adenocarcinoma de cérvix. A la vista de los resultados, se programa una cirugía la semana siguiente. Al disponer del resultado de la biopsia y dada la edad de la paciente se realiza una $T R$ vaginal asistida por laparoscopia con linfadenectomía pélvica, con 1,5 cm de vagina y seccionando el cérvix a $1 \mathrm{~cm}$ del istmo uterino. La anatomía patológica concluye que se trata de un tumor cervical de $9 \mathrm{~mm}$ con invasión de 4 mm en el labio posterior del cérvix y unión escamocolumnar con márgenes laterales libres. 
Los ganglios linfáticos son negativos por lo que se clasifica de estadio IB1. La histología confirma que es un adenocarcinoma mucosecretor cervical. En el mismo acto quirúrgico, tras realizar la TR, se coloca un cerclaje permanente con sutura no reabsorbible de hilos de etilón en cérvix.

A los 33 años, la paciente se embaraza de forma espontánea. El curso del embarazo transcurre normal con biometrías fetales acordes por control ecográfico. En la semana 33, la gestante acude a urgencias por rotura prematura de membranas y se le realiza una cesárea y previa maduración pulmonar con corticoides, después de lo cual nace un varón de 2.950 gramos con un Test de Apgar de 9/9 y un pH en arteria de 7,29 y en vena de 7,23. En cuanto al posoperatorio, este transcurre asintomático.

A los 34 años, la paciente en mención vuelve a embarazarse de forma espontánea. En la semana $22+6$, ingresa en el hospital por rotura prematura de membranas. En la exploración vaginal, el cuello uterino está adelgazado con una medida de $25 \mathrm{~mm}$ y el cerclaje no está a tensión. Por su parte, los cultivos vaginales son negativos. Los análisis sanguíneos al ingreso muestran 9.960 leucocitos, con una neutrofilia de 73,3\% y una proteína C reactiva (PCR) de $4,09 \mathrm{mg} / \mathrm{L}$. La ecografía evidencia un oligoamnios moderado con una biometría fetal de una semana menor que amenorrea, pero con un feto hemodinámicamente estable. En consecuencia, se instaura un tratamiento antibioterápico con ampicilina $500 \mathrm{mg} / 8$ horas y gentamicina $80 \mathrm{mg} / 8$ horas. Igualmente, se realiza maduración pulmonar con dos dosis de un corticoide intramuscular (betametasona $12 \mathrm{mg} / 24$ horas). Además, se instaura un control diario de constantes vitales maternas y de viabilidad fetal. Los análisis sanguíneos se llevan a cabo cada 48 horas. En la semana 23+4, los leucocitos aumentan a 14.000 con $82,8 \%$ de neutrófilos y la PCR a 7,90 mg/dL. En la semana 23+6, el análisis sanguíneo muestra 18.000 leucocitos con $87 \%$ de neutrófilos y una PCR de 32,59 mg/dl. Ese mismo día, la gestante empieza con fiebre de hasta $39^{\circ} \mathrm{C}$, malestar general y sangrado vaginal. Ante la sospecha de corioamnionitis y desprendimiento prematuro de placenta, se le realiza una cesárea, después de la cual nace un feto varón vivo de 340 gramos con importantes signos de inmadurez que fallece a los pocos minutos de vida.

En el posoperatorio, nuevamente se le administran antibióticos a la paciente, como también ergotamínicos. En el día del alta hospitalaria, el recuento de los leucocitos es de 8.300 con una neutrofilia de 63,5\% y una PCR de 4,1 mg/dl.

\section{METODOLOGÍA}

Se hizo una búsqueda en las bases de datos MEDLINE vía PubMed y Cochrane con las palabras clave "cáncer de cérvix", "traquelectomía radical", "embarazo" y "complicaciones obstétricas". El objetivo fue contestar las preguntas ¿cuánto tiempo debe esperar para embarazarse una paciente a la que se le ha realizado una traquelectomía radical?, ¿qué tasa de infertilidad tiene una paciente tras una TR?, ¿se incrementa el riesgo de complicaciones obstétricas en gestantes sometidas a una TR? y icuáles son las más frecuentes?

\section{RESULTADOS}

Siguiendo la metodología descrita, se encontró un total de 69 artículos, de los cuales se evaluaron 17. De esta última cifra, 11 eran artículos de revisión y el resto artículos de opinión y casos clínicos.

\section{DISCUSIÓN}

No existe un consenso en relación al tiempo que debe esperarse para un embarazo tras una TR. La mayoría de los autores sugieren esperar un período de entre seis meses y un año tras una TR vaginal. ${ }^{2,9,10}$ En los casos de TR abdominal, existe menos experiencia y la recomendación es esperar dos años hasta la concepción. ${ }^{13}$ Nuestro centro aconseja esperar al menos un año hasta el embarazo después de una TR tanto vaginal como abdominal, tal como procedió la paciente del caso descrito.

La esterilidad tras una TR varía entre el 25\% y el 30\%. ${ }^{8,11}$ Según Bernardini et ál, la esterilidad tras 
una TR se debe en un 78\% de los casos a un factor cervical, bien por la estenosis del cérvix uterino, por la disminución del moco cervical o por el cuadro adherencial secundario a la cirugía. ${ }^{14}$ Un pequeño porcentaje de estas pacientes presenta hematómetra y para resolverla se requiere una dilatación del cérvix uterino, en los casos en los que se deje un remanente, o del fondo del saco vaginal, en caso de extirpación total del cuello uterino. ${ }^{11}$ Algunos autores creen que las cicatrices del orificio cervical tras una TR vaginal están relacionadas con el tiempo en que permanecen los catéteres intrauterinos tras la cirugía, que suele variar entre tres días y tres semanas. ${ }^{11}$ En las series descritas de pacientes después de una TR abdominal, la mayoría tienen un patrón menstrual normal a las ocho semanas de la cirugía. ${ }^{13}$ Por otra parte, en pacientes que presentan dificultad para embarazarse tras una TR, se debería realizar un estudio completo de esterilidad ya que pueden necesitar de técnicas de reproducción asistida, tales como la estimulación ovárica, la inseminación intrauterina o la fecundación in vitro. En la serie de diez pacientes publicada por Olawaiye et ál, sólo tres consiguieron embarazarse tras la TR abdominal, una de forma espontánea, otra mediante fecundación in vitro y la tercera tras inducción de la ovulación e inseminación intrauterina.${ }^{15}$ En nuestro caso, la paciente se embarazó dos veces de manera espontánea.

Durante el embarazo, las complicaciones obstétricas aumentan. En el primer trimestre, la proporción de abortos tras una TR vaginal es 16\%-20\% mayor que en la población general. ${ }^{5,8,12} \mathrm{El}$ aborto o pérdida fetal en el segundo trimestre es el doble en las gestantes tras una TR vaginal en comparación con la población general (8\%-10\% versus 4\%). ${ }^{5,8,11}$ Estas pérdidas fetales en el segundo trimestre se deben, en la mayoría de los casos, a infecciones ascendentes desde la vagina hasta la cavidad uterina, facilitadas por el acortamiento cervical y la ausencia de tapón mucoso, lo cual produce corioamnionitis y rotura prematura de membranas. ${ }^{8,12}$ Plante et ál abogan por dejar al menos $1 \mathrm{~cm}$ de cérvix in situ, lo que explicaría su reducida tasa de abortos en el segundo trimestre en su serie de 72 pacientes tras TR vaginal. ${ }^{9}$

El parto pretérmino se incrementa en las gestantes que han sido sometidas a una TR vaginal, con una prevalencia de entre $20 \%$ y $30 \% .^{5,10,11}$ Los mecanismos del parto pretérmino son similares a los expuestos para el aborto del segundo trimestre. ${ }^{8,12}$ A la vista de estos resultados obstétricos, muchos autores proponen un seguimiento exhaustivo de estas gestantes en unidades de medicina maternofetal y tocología de alto riesgo, donde se les realizarán cervicometrías ecográficas de forma seriada desde el inicio del embarazo. ${ }^{5,16}$ En los casos de cérvix muy acortado, debería plantearse la realización de un cerclaje, si no se ha realizado de forma profiláctica tras la TR. ${ }^{7}$ Otras medidas preventivas propuestas en el embarazo de estas gestantes son la administración de antibióticos y de corticoides para maduración pulmonar. ${ }^{17}$ En cualquier caso, creemos que hay que individualizar cada situación. Aunque un 40\% de las gestaciones tras una TR llegan a término ( $>37$ semanas), el 60\% restante presentan abortos o partos prematuros. ${ }^{5,8}$ El parto vaginal sólo se permite en aquellas pacientes que no tengan cerclaje permanente. ${ }^{5,8}$ Asimismo, el período de dilatación de las pacientes con una TR suele ser más largo debido a la estenosis cervical, por lo que en muchos casos se recurre al empleo de prostaglandinas vaginales para facilitar la maduración cervical. ${ }^{17}$ En los casos de cerclaje permanente, se deberá programar una cesárea con el feto a término. ${ }^{7}$

En cuanto a la recurrencia del cáncer de cérvix, se publicó una revisión realizada en seis centros hospitalarios con un total de 319 casos. En dicha revisión, después de una media de seguimiento de 44 meses desde la cirugía se encontró 4,1\% de recurrencias del tumor (en parametrios, pared pélvica, pelvis y ganglios linfáticos a distancia) y 2,5\% de muertes debidas a la enfermedad. ${ }^{12}$

\section{CONCLUSIÓN}

En conclusión, las TR vaginales o abdominales son dos técnicas alternativas a tener en cuenta en el 
tratamiento de estadios precoces del carcinoma cervical en mujeres jóvenes con deseo genésico. Asimismo, el pronóstico obstétrico de las pacientes sometidas a TR es bueno a pesar del aumento de complicaciones obstétricas durante el embarazo. En este sentido, aproximadamente la mitad de las gestantes sometidas a una TR tienen resultados obstétricos favorables con respecto a la población general.

\section{CONFIDENCIALIDAD}

En todo momento se mantiene la confidencialidad de la paciente. Ella autoriza la publicación del caso, en el que no aparecen reflejados sus datos personales.

\section{AGRADECIMIENTOS}

Quiero expresar mi agradecimiento a todo el equipo multidisciplinar que trató a la paciente por el caso referido: a la Unidad de Oncología Ginecológica, a la Unidad de Suelo Pélvico, a la Unidad de Fisiopatología fetal, a la Unidad de Tocología de alto riesgo y al equipo del paritorio del Hospital La Paz en Madrid (España).

\section{REFERENCIAS}

1. Parkin DM, Bray F, Ferlay J, Pisani P. Global Cancer Statistics, 2002. CA Cancer J Clin 2005;55:74-108.

2. Burnett AF. Radical trachelectomy with laparoscopic lymphadenectomy: review of oncologic and obstetrical outcomes. Curr Opin Obstet Gynecol 2006;18:8-13.

3. Sonoda Y, Abu-Rustum NR, Gemignani ML, Chi DS, Brown CL, Poynor EA, et al. A fertility-sparing alternative to radical histerectomy: how many patients may be eligible? Curr Opin Oncol 2004;95:534-8.

4. Dargent D, Brun JL, Roy M, Remy I. Pregnancies following radical trachelectomy for invasive cervical cancer. Gynecol Oncol 1994;52:105.

5. Plante M. Vaginal radical trachelectomy: an update. Gynecol Oncol 2008;111:S105-10.
6. Ramírez PT, Schmeler KM, Soliman PT, Frumovitz M. Fertility preservation in patients with early cervical cancer: Radical trachelectomy. Gynecol Oncol 2008; 110:S25-8

7. Gien LT, Covens A. Fertilty-sparing options for early stage cervical cancer. Gynecol Oncology 2010;117:350-2.

8. Beiner ME, Covens A. Surgery insight: radical vaginal trachelectomy as a method of fertility preservation for cervical cancer. Nat Clin Pract Oncol 2007;4:353-61.

9. Boss EA, van Golde RJ, Beerendonk CC, Massuger LF. Pregnancy alter radical trachelectomy: a real option? Gynecol Oncol 2005;99:S152-6.

10. Jolley JA, Battista L, Wing DA. Management of pregnancy after radical trachelectomy: case reports and systematic review of literature. Am J Perinatol 2007;24:531-9.

11. Plante M, Renaud MC, François H, Roy M. Vaginal radical trachelectomy: an oncollogically safe fertility-preserving surgery. An updated surgery of 72 cases and review of the literature. Gynecol Oncol 2004;94:614-23.

12. Schlaerth JB, Sitos NM, Schlaerth AC. Radical trachelectomy and pelvic lymphadenectomy with uterine preservation in the treatment of cervical cancer. Am J Obstet Gynecol 2003;188:29-34.

13. Ungár L, Pálfalvi L, Hogg R, Siklós P, Boyle DC, Del Priore G, et al. Abdominal radical trachelectomy: a fertility-preserving option for women with early cervical cancer. BJOG 2005;112:366-9.

14. Olawaiye A, Del Carmen M, Tambouret R, Goodman A, Fuller A, Duska LR. Abdominal radical trachelectomy: success and pitfalls in a general gynecologic oncology practice. Gynecol Oncol 2009;112:506-10.

15. Petignat P, Stan C, Megevand E, Dargent D. Pregnancy after trachelectomy: a high risk condition of preterm delivery. Report of a case and review of the literature. Gynecol Oncol 2004;94:575-7.

16. Shepherd JH, Mould T, Oram DH. Radical trachelectomy in early stage carcinoma of the cervix: outcome as judged by recurrence and fertility rates. BJOG 2001;108: 882-5.

17. Bernardini M, Barret J, Seaward G, Covens A. Pregnancy outcome in patients after radical trachelectomy. Am J Obstet Gynecol 2003;189:1378-82. 